\title{
Locus Ceruleus and Anterior Cingulate Cortex Sustain Wakefulness in a Novel Environment
}

\author{
Heinrich S. Gompf, Christine Mathai, Patrick M. Fuller, David A. Wood, Nigel P. Pedersen, Clifford B. Saper, and Jun Lu \\ Department of Neurology, Program in Neuroscience and Division of Sleep Medicine Harvard Medical School, Beth Israel Deaconess Medical Center, \\ Boston, Massachusetts 02115
}

Locus ceruleus (LC) neuronal activity is correlated with the waking state, yet LC lesions produce only minor alterations in daily wakefulness. Here, we report that sustained elevations in neurobehavioral and EEG arousal in response to exposure to an environment with novel stimuli, including social interaction, are prevented by selective chemical lesions of the LC in rats. Similar results are seen when the anterior cingulate cortex (ACC), which receives especially dense LC innervation, is selectively denervated of LC input or is ablated by the cell-specific neurotoxin ibotenic acid. Anterograde tracing combined with tyrosine hydroxylase immunohistochemistry demonstrates ACC terminals in apposition with the distal dendrites of LC neurons. Our data implicate the ACC as both a source of input to the LC as well as one of its targets and suggests that the two structures engage in a dialog that may provide a critical neurobiological substrate for sustained attention.

\section{Introduction}

Single-unit recordings in rats and monkeys demonstrate a strong correlation between behavioral state and locus ceruleus (LC) neuronal activity. LC activity is highest during wakefulness, progressively lower during non-rapid eye movement (REM) sleep, and nearly absent during REM sleep (Aston-Jones and Bloom, 1981; Takahashi et al., 2010). Electrical and pharmacological stimulation or inhibition of the region containing the LC promotes or inhibits wakefulness, respectively (Berridge and Foote, 1991; Foote et al., 1991; Berridge et al., 1993; Berridge and Waterhouse, 2003), but both the drugs and current may spread beyond the LC to other structures in the dorsolateral pons that may also play important roles in arousal and wake-sleep control, such as the parabrachial nucleus and the preceruleus region ( $\mathrm{Lu}$ et al., 2006). Chemical or genetic lesions limited to the LC produce only small effects on the amount or timing of wakefulness (Matsuyama et al., 1973; Lidbrink, 1974; Roussel et al., 1976; Monti et al., 1988; González et al., 1998; Ouyang et al., 2004; González and Aston-Jones, 2006), but this has been attributed to redundancy of networks comprising the arousal system or compensatory upregulation of noradrenergic transmission from the few remaining LC neurons following incomplete lesions (Lidbrink, 1974). Thus, although the role of the LC in arousal remains unclear, LC neurons do respond vigorously to novel environmental stimuli (Vankov et al., 1995), and pharmacological inhibition of LC transmission does impair exploratory behavior (Sara et al., 1995). We therefore hypothesized that the LC may

Received June 14, 2010; revised Sept. 3, 2010; accepted Sept. 10, 2010.

This research was supported by Public Health Service Grants HL060292, NS051609, and AG000222. We thank Quan Ha for technical assistance.

Correspondence should be addressed to Dr. Heinrich Gompf, Department of Neurology, Beth Israel Deaconess Medical Center, 3 Blackfan Circle, Boston, MA 02115. E-mail: hgompf@bidmc.harvard.edu.

DOI:10.1523/JNEUROSCI.3037-10.2010

Copyright $\odot 2010$ the authors $\quad$ 0270-6474/10/3014543-09\$15.00/0 primarily support arousal in circumstances requiring sustained alertness in the presence of novel stimuli (Léger et al., 2009).

Widespread LC innervation provides the cerebral cortex, including the anterior cingulate cortex (ACC), with noradrenaline during times of elevated arousal and focused attention (AstonJones and Cohen, 2005). Recent models emphasize descending projections from the ACC and prelimbic and infralimbic cortexes back to the LC, which may be involved in volitional control of waking (Aston-Jones and Cohen, 2005). Whereas the ACC, like the LC, is involved in a wide variety of species- and task-specific behaviors requiring vigilance and arousal (Kolb, 1990), only sparse cortical inputs to the LC core have been found in rats (Cedarbaum and Aghajanian, 1978; Aston-Jones et al., 1986; Hurley et al., 1991; Luppi et al., 1995), although they are suggested by preliminary data to be in monkeys (Aston-Jones and Cohen, 2005). However, numerous inputs to distal LC dendrites modulate LC activity (Van Bockstaele et al., 1996), and these are a target of ACC axons in monkeys (Aston-Jones and Cohen, 2005) and may receive inputs from the infralimbic cortex in rats (Hurley et al., 1991). We therefore examined both the role played by the LC and ACC in sustained neurobehavioral and EEG arousal in the presence of novel stimuli, and the connections between these two areas, in rats.

\section{Materials and Methods}

Animals. Adult male Sprague Dawley rats (275-300 g; Harlan) were individually housed and had ad libitum access to food and water. All animals were housed under controlled conditions (12 h light/dark cycle, starting at 7:00 A.M.; 100 lux) in an isolated ventilated chamber maintained at $20-22^{\circ} \mathrm{C}$. All protocols were approved by the Institutional Animal Care and Use Committees of Beth Israel Deaconess Medical Center and Harvard Medical School.

Surgery. Under chloral hydrate anesthesia ( $7 \%$ in saline; $350 \mathrm{mg} / \mathrm{kg}$ ), a fine glass pipette for the delivery of either saline, $0.25-1 \mu \mathrm{g}$ of saporinconjugated anti-dopamine- $\beta$-hydroxylase antibody ( $\alpha$-DBH-SAP), $100-150 \mathrm{nl}$ of $60 \mathrm{mg} / \mathrm{ml}$ ibotenic acid, 6-OHDA, $10-15 \mathrm{nl}$ of $0.1 \%$ chol- 
era toxin $\mathrm{b}(\mathrm{CTb})$, or 3-10 $\mathrm{nl}$ 10\% biotinylated dextranamine was lowered to a precalculated target in the lateral ventricle $[\alpha$-DBH-SAP; coordinates: 0.25 anteroposterior (AP), 1.2 mediolateral (ML), 3.5 dorsoventral (DV)], bilaterally into the anterior cingulate cortex (6-OHDA, ibotenic acid, biodextran; coordinates: $1.25 \mathrm{AP}, \pm 0.5 \mathrm{ML}, 1.0 \mathrm{DV})$ or unilaterally and bilaterally into the LC (6-OHDA, CTb; coordinates: -9.7 AP, 1.3 ML, 6.4 DV) based on the rat atlas of Paxinos and Watson (1998) and the solution was injected by an air pressure system. Tracer injections targeted the LC itself; lesions of the LC were performed by injection of $\alpha$-DBH-SAP into the lateral ventricle as described by Wrenn et al. (1996) or 6-OHDA injection directly into the LC as described by Cirelli and Tononi (1996). Tracer injections and lesions of the ACC were targeted in the anterior cingulate cortex and were later confirmed to also involve portions of the premotor and prelimbic areas. Lesions were later verified by tyrosine hydroxylase (TH) and $\mathrm{DBH}$ immunostaining and/or by Nissl staining. None of the injections resulted in morbidity or mortality, and animals remained healthy throughout the experimental period.

EEG/EMG recording and sleep analysis. After animals were anesthetized with chloral hydrate and injections were performed as described above, the skulls were exposed. Four EEG screw electrodes were implanted into the skull, two in the frontal and two in the parietal bones, and two flexible EMG wire electrodes were placed into the neck muscles. The free ends of the leads were fit into a socket that was attached to the skull with dental cement. Two weeks after surgery, the sockets were connected via flexible recording cables and a commutator to a Grass polygraph and computer, and signals were digitized using a Dell PC running the Sleep Sign recording system (Kissei Comtec). The EEG/EMG was recorded for $48 \mathrm{~h}$ at the end of the second week after surgery.

Wake-sleep states were automatically scored and manually checked in $12 \mathrm{~s}$ epochs on the digitized EEG/EMG. Wakefulness was identified by the presence of a desynchronized EEG and phase EMG activity. Additionally, wakefulness was distinguished from sleep states by use of video monitoring. Non-rapid eye movement (NREM) sleep consisted of a high amplitude slow-wave EEG together with a low EMG tone relative to wake. REM sleep was identified by the presence of regular theta EEG activity coupled with low EMG tone relative to NREM sleep. The amount of time spent in wake, NREM sleep, and REM sleep was determined for each 35 min period.

Environmental complexity. Rats were paired with unlesioned partners. Within the sleep recording chamber, individual cages were placed next to each other and the tops were removed, permitting the animals to move freely from cage to cage. After a $30 \mathrm{~min}$ period during which animals were allowed to approach each other in the absence of other distractions, they were exposed to the same series of novel objects placed in both cages every $70 \mathrm{~min}$. Objects were chosen on the basis of maximizing interactive value for the rat (van Praag et al., 2000; Nithianantharajah and Hannan, 2006), including those with varying textures (hard, knobby objects like plastic building blocks and soft rubbery objects), and ones involving minor exploration and tasks such as treats hidden in plastic cones with holes large enough for a rat to enter the top, and green shredded paper (artificial grass) that could be used for bedding. The amount of time between object presentations $(70 \mathrm{~min}$ ) was optimized in preliminary experiments to provide a control rat with the maximum amount of sustained arousal with the minimum amount of investigator interference. The total exposure time to this complex environment lasted $5 \mathrm{~h}$. Following at least $2 \mathrm{~d}$ habituation to the sleep recording chamber, EEG/EMG and video were recorded for at least $24 \mathrm{~h}$ of baseline sleep/wake (without toys) before the environmental complexity procedure commenced (Zeitgeber time (ZT) 6-11, or 6-11 h after lights on). Novel objects were presented as sets (Fig. 1A) to all animals at the same time relative to the start of the experiment. During the entire time, although animals were free to sleep or stay awake, unlesioned animals stayed awake almost continuously. Neither social novelty alone ( $57 \pm 2.5 \%$ wakefulness) nor novel objects presented every $70 \mathrm{~min}$ alone (51.6 $\pm 3.0 \%$ wakefulness) was able to achieve this, so the combination of both was used for these experiments. Beyond that, exposing animals to the social novelty while presenting the same objects every $70 \mathrm{~min}$ (a disturbance control) also did not evoke as much wakefulness in saline-injected animals $(73.3 \pm 7.8 \%$ wakefulness), suggesting that novelty in the objects is an important factor in maintaining wakefulness under these conditions. Animals spent the majority $(79.5 \pm 2.6 \%)$ of their time interacting with the novel features of their environment (objects, the cage mate, climbing onto and investigating the top of the open cages) and spent the rest of the time grooming, eating, drinking, and not interacting with the presented novelty. In between experiments, objects that remained intact were cleaned in a diluted bleach solution and rinsed numerous times with warm water, whereas those objects that had visible tooth marks were replaced with new objects. Artificial grass was never reused.

Immunohistochemistry. Animals were deeply anesthetized with isoflurane and then perfused with $50 \mathrm{ml}$ of saline followed by $500 \mathrm{ml}$ of $10 \%$ formalin through the heart. The brains were removed, postfixed for $2 \mathrm{~h}$ in $10 \%$ formalin, and then equilibrated in $20 \%$ sucrose in PBS containing azide overnight. The brains were sectioned on a freezing microtome at 40 $\mu \mathrm{m}$ into four series. Sections were washed in $0.1 \mathrm{M} \mathrm{PBS}, \mathrm{pH} 7.4$ (two changes), and then incubated in the primary antiserum for $1 \mathrm{~d}$ at room temperature. For c-Fos, we used a rabbit polyclonal antiserum (AB5, 1:50,000; Oncogene Sciences) against residues 4-17 from human c-Fos. This antiserum stains only the nuclei of neurons based on recent activity patterns (Gaus et al., 2002; Lu et al., 2002). The TH antibody (1:20,000, catalog no. 22941; ImmunoStar) used was a mouse monoclonal antibody raised against $\mathrm{TH}$ isolated from rat PC12 cells. Western blots of HEK293 cells transfected with TH isoform 2 showed a single specific band at 60 $\mathrm{kDa}$ that was not present in untransfected cells. This antibody stains a pattern of neuronal morphology and distribution identical to previous reports (Takada et al., 1990; Sauer and Oertel, 1994). Sections were then washed in PBS and incubated in biotinylated secondary antiserum (against appropriate species IgG; 1:1000 in PBS) for $1 \mathrm{~h}$, washed in PBS, and incubated in avidin-biotin-horseradish peroxidase conjugate (Vector Laboratories) for $1 \mathrm{~h}$. Sections were then washed again and incubated in a $0.06 \%$ solution of 3,3-diaminobenzidine tetrahydrochloride (DAB; Sigma) plus $0.02 \% \mathrm{H}_{2} \mathrm{O}_{2}$. The sections were stained brown with DAB only or black by adding $0.05 \%$ cobalt chloride and $0.01 \%$ nickel ammonium sulfate to the DAB solutions. For confocal microscopy, biotinylated dextranamine was visualized using streptavidin and Alexa Fluor 546 conjugate (1:500; Invitrogen), and TH was visualized using an Alxea Fluor 488 donkey anti-mouse secondary antibody (1:500; Invitrogen). Z-stack sections of the slice were $0.75 \mu \mathrm{m}$ thick and the total section thickness was $15 \mu \mathrm{m}$.

Cell counting. Stereological counting of noradrenergic cells or c-Fos staining in these studies was precluded because the antibodies used did not penetrate full section thickness. We therefore used a post hoc Abercrombie correction for cell size. Counters were blinded to the treatments in each case. Counts of c-Fos-positive neurons were performed similarly for each brain region. Three slices (120 $\mu \mathrm{m}$ apart) from each animal were chosen based on anatomical landmarks to ensure equivalent planes were counted for each animal section. For cortical regions, under $25 \times$ magnification, a $1.0 \times 1.0 \mathrm{~mm}$ box was placed bilaterally inside each defined area (cingulate, frontal, insular) (Paxinos and Watson, 1998) for counting. Approximate locations of the $1.0 \times 1.0 \mathrm{~mm}$ counting boxes (e.g., at the caudal-most level) are depicted in the inset of the supplemental Figure, available at www.jneurosci.org as supplemental material. LC c-Fos expression was counted in the areas outlined by Watson and Paxinos (1998). Counts for each area from all three sections were averaged. For TH neuronal counts, three sections containing the densest part of the individual brain regions (LC, A2, C2, A5, A7, A1), as previously described (Paxinos and Watson, 1998), were counted. Each labeled cell with a visible nucleus was counted, an Abercrombie correction was applied, and the mean number of cells on each side of the brain for each section is reported.

Data analysis. Student's $t$ tests were performed in Microsoft Excel. ANOVA analyses were performed on GraphPad Prism (version 4.0b for Macintosh; GraphPad Software). Total percentage wake over the $5 \mathrm{~h}$ test period compared with the control rest period $24 \mathrm{~h}$ prior was analyzed with a one-way ANOVA followed by a Tukey's post hoc pairwise comparison for each animal. Total percentage wake over the $5 \mathrm{~h}$ test period (in 35 min bins) was analyzed with two-way (time and treatment) ANOVA, followed by Bonferroni's post hoc multiple-comparison test if the main analysis showed a statistical significance. 

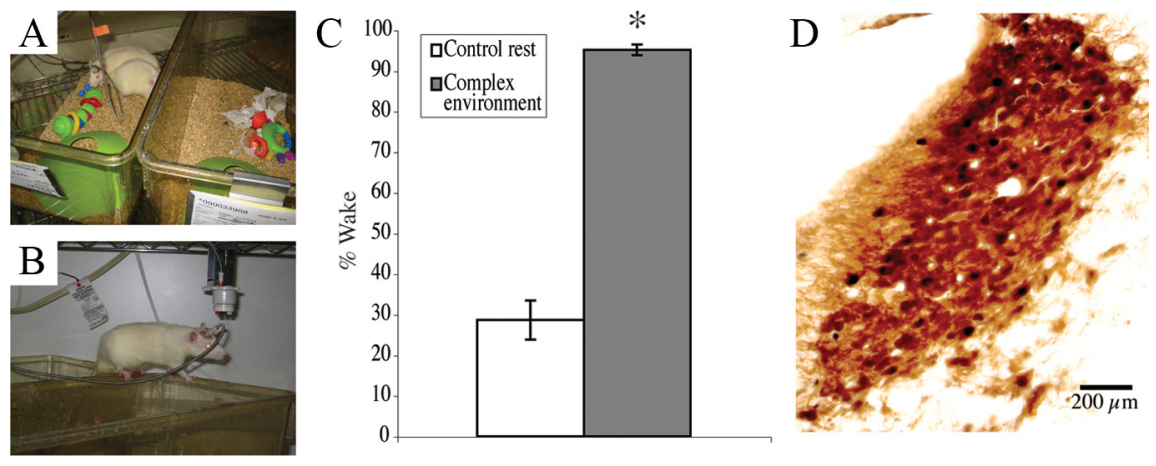

Figure 1. Wakefulness in a complex environment. $\boldsymbol{A}, \boldsymbol{B}$, Example of complex environment. Two rats were paired by bringing together two regular housing cages with tops removed $(\boldsymbol{A})$ so that they were free to move between cages $(\boldsymbol{B})$ and interact. Novel objects were added - baby rattles and plastic cones are shown in $\boldsymbol{A}$ - every 70 min for $5 \mathrm{~h}$ to arouse curiosity and exploration. $\boldsymbol{C}$, Animals spent significantly $\left({ }^{*} p=1 \times 10^{-7}\right)$ more time awake in this environment than during the same period $24 \mathrm{~h}$ prior in their home cages. $\boldsymbol{D}$, Double immunohistochemistry for tyrosine hydroxylase (brown cytoplasm) and c-Fos (black nuclei) at the level of the LC. LC c-Fos expression, usually absent during baseline wakefulness, increased dramatically during novelty exposure.

\section{Results}

A complex, novel environment enhances wake drive

Placing rats into a novel environment, either with novel objects (baby toys) or with cagemates, or with both, was not sufficient to cause sustained wakefulness beyond $\sim 1 \mathrm{~h}$ during the light period (ZT6-11). However, if we changed the toys every hour or so, the animals would remain awake and interact with the novel features of their environment almost continuously for up to $5 \mathrm{~h}$, indicating that novelty plays a major role in keeping the animals awake during what should be their sleep period. When we combined these stimuli during the $5 \mathrm{~h}$ period, animals spent $333 \%$ more time awake compared with baseline $(95.1 \pm 1.3 \%$ vs $28.6 \pm 4.8 \%$ wakefulness in the equivalent period $24 \mathrm{~h}$ prior, $p=1 \times 10^{-7}, n=6$ ) (Fig. $1 C$ ). c-Fos expression was evaluated in the LC at the end of this period. Typically, in our laboratory, few LC neurons expressed c-Fos during spontaneous wakefulness [for instance, 0 cells per side in both spontaneous wakefulness and sleep was reported by Lu et al. (2008)], yet here we found widespread c-Fos expression under the novelty condition ( $126 \pm 5.3$ cells per side, $n=5$ ) (Fig. $1 D)$, which prompted us to hypothesize that the LC plays a role in the maintenance of arousal under these conditions.

To control for stress caused by this procedure, we measured the numbers of cFos-immunoreactive neurons in the paraventricular nucleus of the hypothalamus after the procedure, compared with other manipulations that cause rats to maintain wakefulness during the sleep period. After the novelty procedure, there were a mean of $12.6 \pm 0.9$ Fos-positive neurons per section versus $57.8 \pm 5.8$ neurons per section after $5 \mathrm{~h}$ of gentle handling typically used to cause the same amount of sleep loss $(n=4$ animals each, three slices counted bilaterally for each animal). The Fos counts in the paraventricular nucleus after exposure to the novel environment were similar to the counts Cano et al. (2008) observed in animals that had spent the majority of $5.5 \mathrm{~h}$ asleep following transfer into a clean cage at the same time of day. Considering that our animals were awake for $5 \mathrm{~h}$, the low c-Fos counts suggest that the novel environment presents a minimal degree of stress.

\section{LC lesions compromise the maintenance of wakefulness in a complex, novel environment}

Saporin conjugated to an antibody specific to the noradrenaline synthesizing enzyme, $\alpha$-DBH-SAP $(0.25-1 \mu \mathrm{g})$ was injected into the lateral ventricle. This drug selectively targets noradrenergic neurons and, when injected in this way, has been reported to eliminate $>95 \%$ of LC neurons while sparing other noradrenergic or adrenergic cell groups (Wrenn et al., 1996) (Fig. 2). In our sections, we counted $6.8 \pm 0.4$ and $9.3 \pm 1.3 \mathrm{TH}$-positive LC neurons remaining per side in 0.5 and $1.0 \mu \mathrm{g}$ of $\alpha$-DBH-SAPinjected animals, respectively. Counting LC neurons in $40-\mu \mathrm{m}-$ thick $\mathrm{TH}$-stained sections from saline-injected control animals was not feasible (because the neuronal cell bodies were tightly packed together and overlapped one another in different tissue planes), but in Nissl-stained sections from the same animals we found 246.0 \pm 7.5 LC neurons per section, indicating that we had eliminated at least $95 \%$ of LC neurons. We were also able to
Figure 2. $A-L$, A series of photomicrographs showing loss of TH-immunoreactive neurons in the $L C$ at three levels, after injection of saline $(\boldsymbol{A}-\boldsymbol{C})$ or increasing doses $(\boldsymbol{D}-\boldsymbol{F}, 0.25$ $\mu \mathrm{g} ; \mathbf{G}-\mathbf{I}, 0.5 \mu \mathrm{g} ; \boldsymbol{J}-\boldsymbol{L}, 1 \mu \mathrm{g})$ of $\alpha$-DBH-SAP into the lateral ventricle. Note the substantial reduction in TH-positive $\mathrm{LC}$ neurons in all $\alpha$-DBH-SAP-treated groups relative to control saline injections, and that the cell loss following 0.5 and $1.0 \mu \mathrm{g}$ injections was near maximal. 


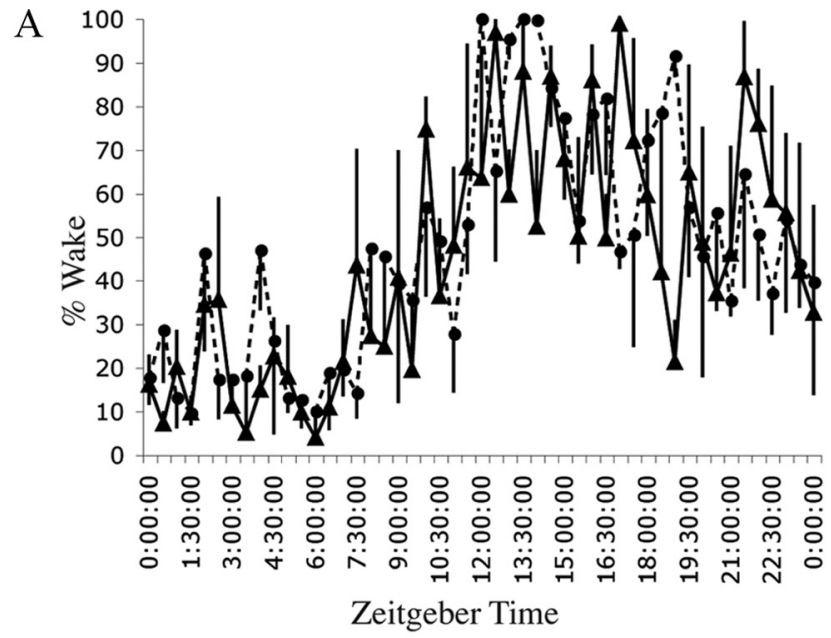

B

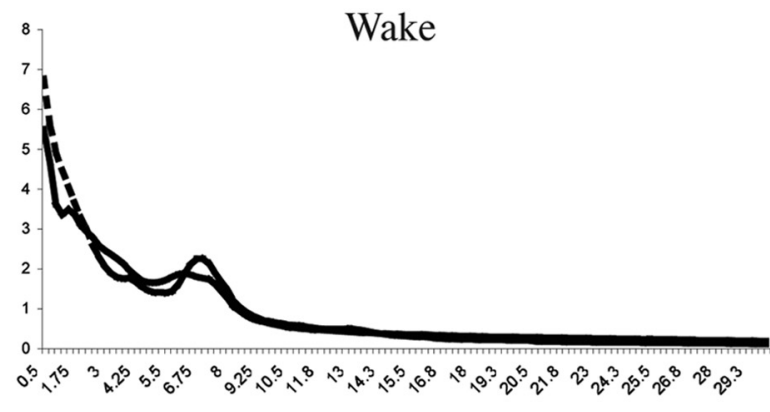

$\mathrm{C}$

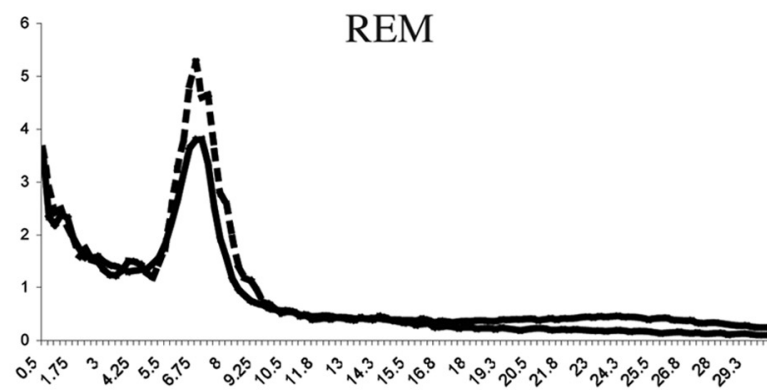

$\mathrm{D}$

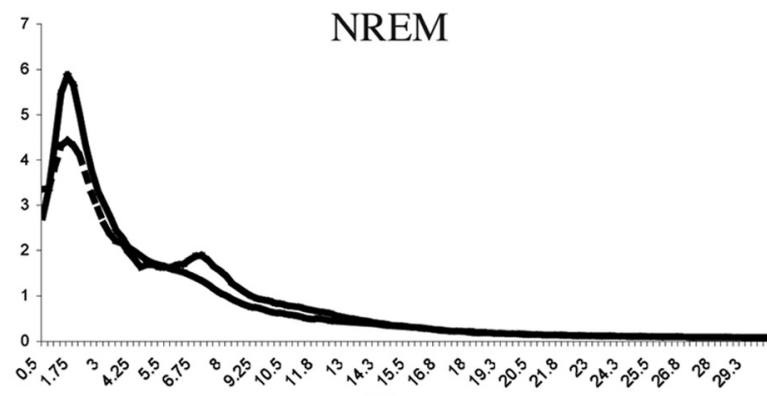

$\mathrm{Hz}$

Figure 3. LC lesions do not affect baseline wakefulness. $A$, Percentage wake time \pm SEM per 30 min period in their home cage on the baseline recording day for intact animals ( $n=3$, solid line) and animals with $\mathrm{LC}$ lesions due to injection of $0.5 \mu \mathrm{g}$ of $\alpha$-DBH-SAP into the lateral ventricle $(n=3$, dashed line). Time is shown with respect to lights on (7 A.M., Zeitgeber time). No significant differences were found ( $p=0.9574, F=0.2115, n=3$ each). $\boldsymbol{B}-\boldsymbol{D}$, Power spectra for control $(n=3$, solid line) and $0.5 \mu \mathrm{g}$ of $\alpha$-DBH-SAP-treated ( $n=3$, dashed line) animals for the wake ( $\boldsymbol{B})$, REM sleep $(\boldsymbol{C}$, and slow-wave sleep (D) stages over frequency ranges from 0.5 to $30 \mathrm{~Hz}$. No significant differences were found at any frequency in any stage (repeated-measures ANOVA, $p>0.9$ for each point).

confirm that the numbers of A1/C1, A2, C2, A5, and A7 neurons were unaffected by this treatment (supplemental Table 1, available at www.jneurosci.org as supplemental material). LC lesions did not affect baseline total wake time per $24 \mathrm{~h}(44.0 \pm 3.7 \%$
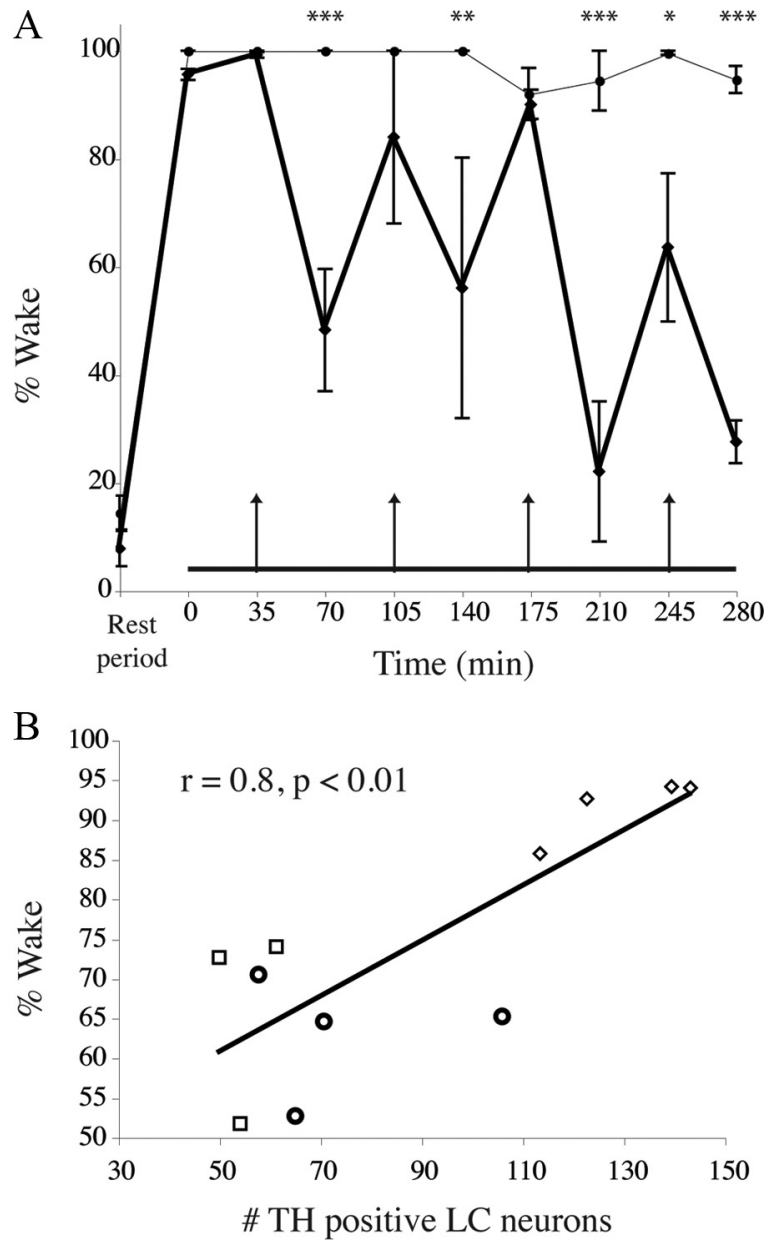

Figure 4. Reduced wakefulness during novelty exposure in LC-lesioned animals compared with saline-injected controls. A, Averaged total wakefulness of $0.5 \mu \mathrm{g}$ of $\alpha$-DBH-SAP-treated animals ( $n=4$, thick line) compared with saline-injected controls (thin line) in 35 min bins over the $5 \mathrm{~h}$ novelty exposure. The first data point represents wakefulness in the $35 \mathrm{~min}$ period just before the complex environment period (horizontal line at bottom). Arrows indicate times of presentation of new sets of novel objects. Note that saline-treated control animals maintain almost full wakefulness, whereas $\alpha$-DBH-SAP-treated animals show increased wakefulness bouts around the time of novel object presentation, which is then markedly decreased in the second half of each $70 \mathrm{~min}$ period. This trend was more pronounced the longer animals were exposed to environmental complexity. Points are means \pm SEM. ${ }^{*} p<0.05$; ${ }^{* *} p<0.01$; ${ }^{* * *} p<0.001$. B , Correlation between the number of $\mathrm{LC}$ neurons and wakefulness in the complex environment. Diamonds, $0.25 \mu \mathrm{g}$ of $\alpha$-DBH-SAP; circles, $0.5 \mu \mathrm{g}$ of $\alpha$-DBH-SAP; squares, $1 \mu \mathrm{g}$ of $\alpha$-DBH-SAP.

saline vs $47.8 \pm 3.7 \%$ for $0.5 \mathrm{mg}$ of $\alpha$-DBH-SAP, $p=0.9574, F=$ $0.2115, n=3$ for each group, repeated-measures ANOVA comparing each time point) (Fig. $3 A$ ) or from ZT6 $-11(28.6 \pm 4.8 \%$ with saline vs $31.0 \pm 5.5 \%$ with $0.25 \mu \mathrm{g}$ of $\alpha$-DBH-SAP, $p=0.4$; $38.5 \pm 8.8 \%$ with $0.5 \mu \mathrm{g}, p=0.2 ; 32.4 \pm 1.8 \%$ wakefulness with $1 \mu \mathrm{g}, p=0.3)$. LC lesions also did not affect EEG power spectra compared with controls (repeated-measures ANOVA comparing each $0.25 \mathrm{~Hz}$ frequency bin for each stage: wake, REM, and NREM) (Fig. $3 B-D$ ). When exposed to the novel environment from ZT6-11, animals at the higher two doses of $\alpha$-DBH-SAP tended to awaken briefly following presentation of the latest set of novel objects (Fig. 4A, wakefulness "peaks" indicated by arrows) and fall asleep again before presentation of the next set of novel objects, whereas saline-injected control animals remained awake and actively explored the objects until the subsequent set was presented. Compared with sham-lesioned animals $(95.1 \pm 1.3 \%$ 


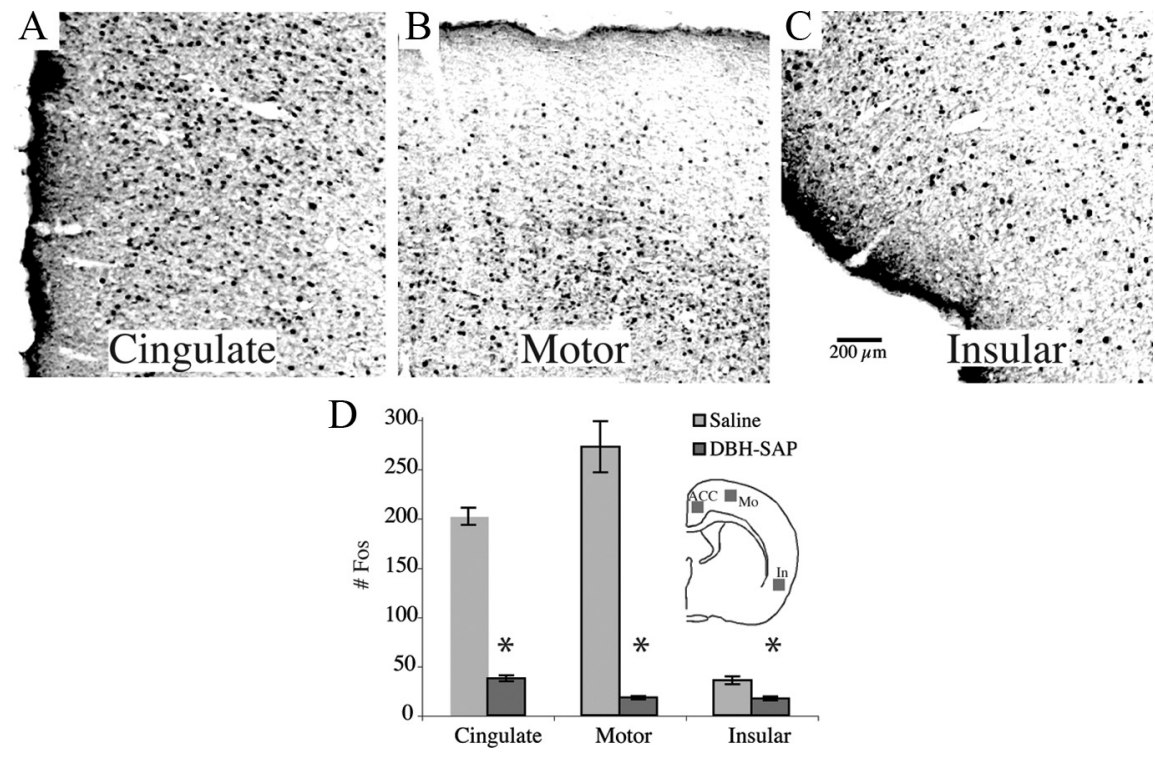

Figure 5. Cortical activation in a complex environment. $\boldsymbol{A}-\boldsymbol{C}$, $\mathrm{C}-$ Fos expression was increased in all cortical areas after $5 \mathrm{~h}$ of sustained wakefulness during novelty exposure, e.g., the anterior cingulate $(\boldsymbol{A})$, motor $(\boldsymbol{B})$, and insular cortices $(\boldsymbol{C})$. D. Elevated cortical c-Fos was maintained throughout the $5 \mathrm{~h}$ period in saline-injected animals. However, $\alpha$-DBH-SAP-treated animals exhibited marked decreases in cortical c-Fos expression. ${ }^{*} p<0.001$. Inset, Approximate location of $1 \times 1 \mathrm{~mm}$ counting boxes. Mo, Motor cortex; In, insular cortex.

Table 1. Numbers of c-Fos-immunoreactive neurons in cortical areas after 2.5 or $5 \mathrm{~h}$ of exposure to novel objects and playmates, in animals injected with saline versus $\alpha$-DBH-SAP

\begin{tabular}{lrrl}
\hline & Cingulate & \multicolumn{1}{l}{ Motor } & Insular \\
\hline Saline $(5 \mathrm{~h})$ & $172.2 \pm 13.0$ & $258.7 \pm 18.2$ & $35.4 \pm 3.9$ \\
$0.5 \mu \mathrm{g} \alpha$-DBH-SAP $(2.5 \mathrm{~h})$ & $238.3 \pm 15.4$ & $247.1 \pm 14.1$ & $34.3 \pm 3.2$ \\
$0.5 \mu \mathrm{g} \alpha$-DBH-SAP $(5 \mathrm{~h})$ & $37.4 \pm 2.8^{*}$ & $17.8 \pm 1.6^{*}$ & $16.9 \pm 1.8^{*}$
\end{tabular}

After $5 \mathrm{~h}$ of environmental enrichment, a significant reduction in cortical c-Fos was seen in LC-lesioned animals compared with saline-injected controls. An ANOVA was performed for each region, indicating differences between groups. Following this, individual $t$ test compared each group to its corresponding saline-injected control group. $n=4$ animals, three slices each, ${ }^{*} p<0.0001$.

wakefulness with saline, $n=6$ ), wakefulness was decreased in $\alpha$-DBH-SAP-treated animals in a dose-dependent manner (89.4 $\pm 4.5 \%$ wakefulness with $0.25 \mu \mathrm{g}, n=4 ; 66.1 \pm 7.3 \%$ with $0.5 \mu \mathrm{g}, n=3 ; 63.2 \pm 3.8 \%$ with $1 \mu \mathrm{g}, n=4$; two-way ANOVA and Bonferroni post hoc tests compared with the saline group, time $\times$ dose interaction, $p<0.0001, F=3.4$ ). A significant correlation was also established between the total amount of wakefulness during the $5 \mathrm{~h}$ exposure to environmental complexity and the estimated total number of remaining $\mathrm{TH}$-positive LC neurons $(r=0.80$; 95\% confidence limits, $0.22-0.92 ; p<0.01)$ (Fig. $4 B$ ), consistent with direct involvement of the LC in maintaining this wakefulness. Hence, in subsequent figures and analyses, the $0.5 \mu \mathrm{g}$ dose was chosen as the representative dose, as it was the lowest dose that resulted in nearly complete elimination of the LC and provided a near-maximal decrease in noveltystimulated wakefulness.

\section{Cortical c-Fos activation}

The LC diffusely innervates the entire cerebral cortex. We therefore measured the number of c-Fos-immunoreactive neurons in three representative areas, the anterior cingulate, motor, and insular cortices as a measure of activation of these regions during the period of exposure to a complex environment. Levels of c-Fos expression were consistent with the observed changes in waking behavior (Fig. $5 A-C$ ), with high c-Fos counts after $5 \mathrm{~h}$ of envi- ronmental complexity in saline-injected control animals, but substantial reduction in animals treated with $0.5 \mu \mathrm{g}$ of $\alpha$-DBHSAP (Table 1, Fig. 5D). The reduction of cortical c-Fos during environmental complexity in animals with LC lesions is consistent with the LC playing a role in sustaining cortical arousal in the complex, novel environment.

However, a previous study (Cirelli et al., 1996) had reported that after unilateral lesions of the LC, there was loss of c-Fos immunoreactivity in the ipsilateral cerebral cortex during wakefulness, suggesting that the low levels of c-Fos expression in the novel environment might be due to the inability of cortical neurons to express c-Fos after noradrenergic denervation. We therefore examined the expression of c-Fos in the cerebral cortex of rats that had bilateral LC lesions (lateral ventricular injections of $\alpha$-DBH-SAP) and which had been awake for $2.5 \mathrm{~h}$ during the first half of the novelty procedure. These animals showed cortical c-Fos expression comparable to control waking animals (Table 1), indicating that LC lesions do not prevent cortical c-Fos expression if animals have been awake. To more closely replicate the method used in the previous study in our complex novel environment, we performed unilateral 6-OHDA lesions of the LC (59.0 \pm 22.2 LC neurons/ lesioned side) (Fig. $6 \mathrm{~A}$ ), producing ipsilateral loss of DBH fibers in the cortex (Fig. $6 \mathrm{~B}$ ). When exposed to the novel environment, these rats maintained wakefulness in the complex novel environment as well as unlesioned animals did (93.25 $\pm 2.7 \%, n=12$ vs $95.1 \pm 1.3 \%$ saline animals as described above, $n=6, p=0.6$ ). Following $5 \mathrm{~h}$ exposure to this environment, c-Fos counts on the side ipsilateral to the lesion were not significantly different from those contralateral to the lesion (cingulate cortex, $115.9 \pm 13.7$ lesioned side vs $117.0 \pm 12.4$ unlesioned side, $p=0.95, n=12$; motor cortex, $91.6 \pm 11.5$ lesioned vs $97.7 \pm 11.2$ unlesioned, $p=$ $0.71, n=12$ ) (Fig. 6C). Thus, the reduced levels of c-Fos expression in the cerebral cortex in animals who failed to maintain wakefulness after LC lesions was due to lack of cortical activation in sleeping animals rather than inability of the cortex to express c-Fos after LC lesions. Furthermore, integrity of the LC on only one side of the brain was sufficient to produce a normal waking response to the novel environment and a comparable c-Fos response in both hemispheres.

\section{The anterior cingulate cortex is involved in sustained arousal} in complex, novel environments, and projects to the LC

Increased c-Fos expression in the cerebral cortex during exploration of the complex environment suggests that the cortex plays an important role in arousal related to novelty. Whereas the noradrenergic LC innervates the entire cortex, the densest terminal field in rats is in the medial prefrontal cortex, including the ACC (see below). Therefore, we postulated that this area might be especially critical in maintaining the observed behavior. To test this hypothesis, we disrupted noradrenergic inputs to the ACC by injecting the ACC with 6-OHDA, which eliminated catecholaminergic axons and terminals in this region, as well as parts of the adjacent premotor and prelimbic areas and caudally in the 

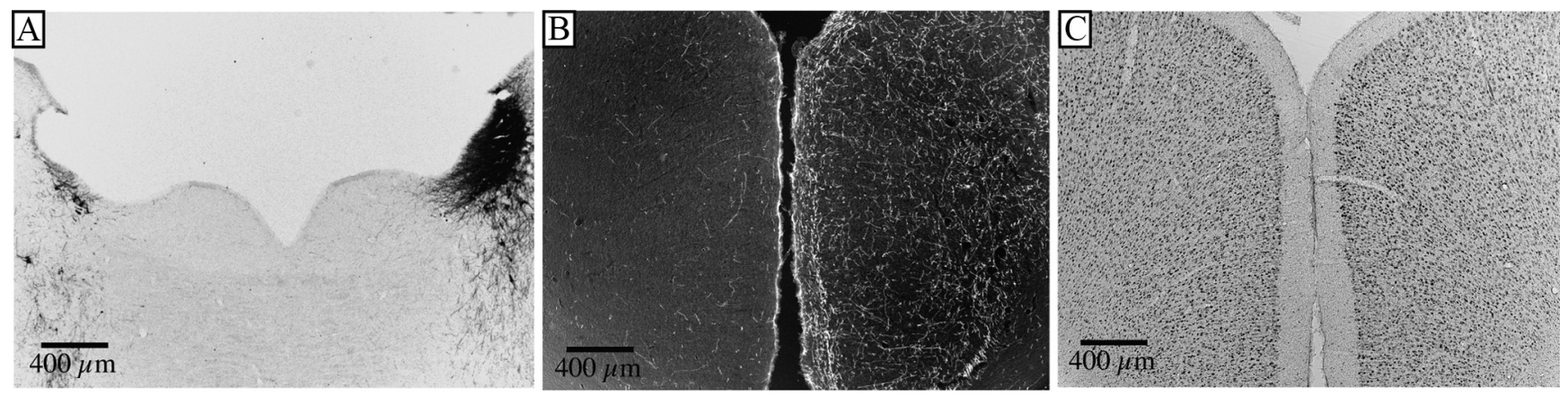

Figure 6. Cortical C-Fos activation is not correlated with noradrenergic fiber loss. $A, B, 6-0 H D A$ unilaterally injected into the $L C$ produced widespread $L C$ neuronal loss $(A)$, accompanied by widespread loss of DBH-immunoreactive fibers in the ipsilateral cerebral cortex (B). $\boldsymbol{C}$, Five hours of sustained wakefulness during novelty exposure evoked widespread c-Fos expression in the ACC, both ipsilateral and contralateral to the LC lesion.

cingulate bundle (Fig. 7A). These animals showed decreased wakefulness in the complex environment, to levels of wakefulness comparable to LC-lesioned animals (65.9 $\pm 5.8 \%$ compared with $66.1 \pm 7.3$ with $0.5 \mu \mathrm{g}, p=0.98, n=4)$ (Fig. $7 C$ ). Animals with 6-OHDA injections in the neighboring motor cortex, without ACC involvement, maintained wakefulness comparable to unlesioned controls $(97.6 \pm 1.1 \%$ compared with $95.1 \pm 1.3, p=0.8$, $n=4)$. c-Fos expression was reduced in both the LC ( $47.1 \pm 3.9$ compared with controls with saline injections into the lateral ventricle, $126 \pm 5.3, p<0.0001)$ and the ACC $(54.5 \pm 5.3 \mathrm{com}-$ pared with saline-injected controls, $172.2 \pm 13.0, p=8.3 \times 10^{9}$ ) following 6-OHDA-mediated eradication of LC terminals in the ACC, although numbers of c-Fos-immunoreactive cells in the ACC were significantly greater than in $\alpha$-DBH-SAP-treated animals (54.5 \pm 5.36 -OHDA vs $37.4 \pm 2.8 \alpha$-DBH-SAP, $p=0.01$, $n=4$ each).

These results suggest that noradrenergic LC inputs to the ACC maintain wakefulness in a complex environment; this finding prompted us to ask whether reciprocal descending projections from the ACC to the LC might sustain LC activity under these conditions. We therefore examined the reciprocal projection from the ACC to the LC by injection of the retrograde tracer $\mathrm{CTb}$ into the LC and the region just medial to it. Numerous retrogradely labeled neurons were found in the cortex, with the most prominent inputs coming from the ACC (Fig. $8 \mathrm{~A}$ ) and prelimbic/infralimbic cortices. Conversely, following injection of the anterograde tracer biotinylated dextranamine into an area encompassing the anterior cingulate and portions of the prelimbic/ infralimbic cortex, small numbers of anterogradely labeled terminals were seen in the LC itself, but much larger numbers were found in the region just medial to the ipsilateral LC, which contains many LC dendrites (Fig. $8 B, C$ ). In examining confocal images of this area, we found numerous appositions between red (anterogradely labeled) terminals and green (TH-immunoreactive) dendrites when examined within a $0.75 \mu \mathrm{m}$ focal plane. These results suggest that inputs to the LC from the ACC may mediate the observed inhibition of sleep drive at times when attentiveness is required.

To test this idea, we also placed bilateral ibotenic acid lesions of the ACC (Fig. $7 B$ ) and recorded the response to the novel environment. These lesions also led to attenuation of wakefulness in the complex environment similar to $\alpha$-DBH-SAP lesions of the LC ( $64.5 \pm 2.4 \%$ wake, $n=4$, compared with $66.1 \pm 7.3$ with 0.5 $\mu \mathrm{g} p=0.9)$ (Fig. 7C). Here too, LC c-Fos was much lower (15.3 \pm 3.1 compared with saline-injected controls, $126 \pm 5.3, p<$ $0.0001, n=4)$. Together, these results suggest a dialog between the ACC and LC that is required for the maintenance of wakefulness under novel conditions during the adverse (sleep phase) of the circadian cycle.

\section{Discussion}

Our results show that the LC appears to be essential for sustained wakefulness in response to novel environmental stimuli. LC innervation of the ACC may be responsible for most of this effect, as either denervating the catecholaminergic input to the ACC or lesions of the ACC have the same effect (reducing wakefulness in the novel environment) as LC lesions. Furthermore, lesions of the ACC reduced c-Fos expression in the LC, suggesting that there is a dialog between these two sites in determining the level of arousal in response to novel environmental stimuli. In essence, this circuit is necessary to prevent what Berridge and España (2000) termed "habituation to novelty." In contrast, the activation of the LC under the novel conditions is not likely to be due to stress, as paraventricular hypothalamic neurons (which are highly responsive to stress) show low levels of c-Fos expression during novelty that are similar to animals sleeping in a new cage during that time of day (Cano et al., 2008).

One possible confound is that the LC lesions may have produced rats that were simply sleepier. However, a previous study of mice with deletion of histidine decarboxylase (HDC) expression also found normal amounts of sleep and wakefulness, but reported that the animals were sleepier, based on having more sleep during the light-dark transition periods and more delta power in the EEG during wakefulness. (Parmentier et al., 2002). Our animals did not have more sleep during the light-dark transition, nor did they have excess delta power in the waking EEG. The $\mathrm{HDC}^{-1-}$ mice also showed a shorter latency to fall asleep when placed in a clean cage during the light period (Parmentier et al., 2002). In our paradigm, in which we changed the novel objects every $70 \mathrm{~min}$, the animals with LC lesions and the intact animals both stayed awake for most of the first $2.5 \mathrm{~h}$, indicating that the rats with LC lesions were not simply sleepier. However, as the animals with LC lesions habituated to the novelty of the situation, they began falling asleep for most of the time between changes of the objects in the last $2.5 \mathrm{~h}$. Our interpretation that LC neurons are responding to the novelty of the situation is consistent with the findings of Léger and colleagues (2009), who reported that placing rats in a novel environment during the light cycle kept them awake for $3 \mathrm{~h}$. Under these conditions, $\sim 30 \%$ of LC neurons showed c-Fos expression, whereas sleep deprivation by itself for a similar period of time did not have this effect. These examples suggest that testing wakefulness in novel conditions may 

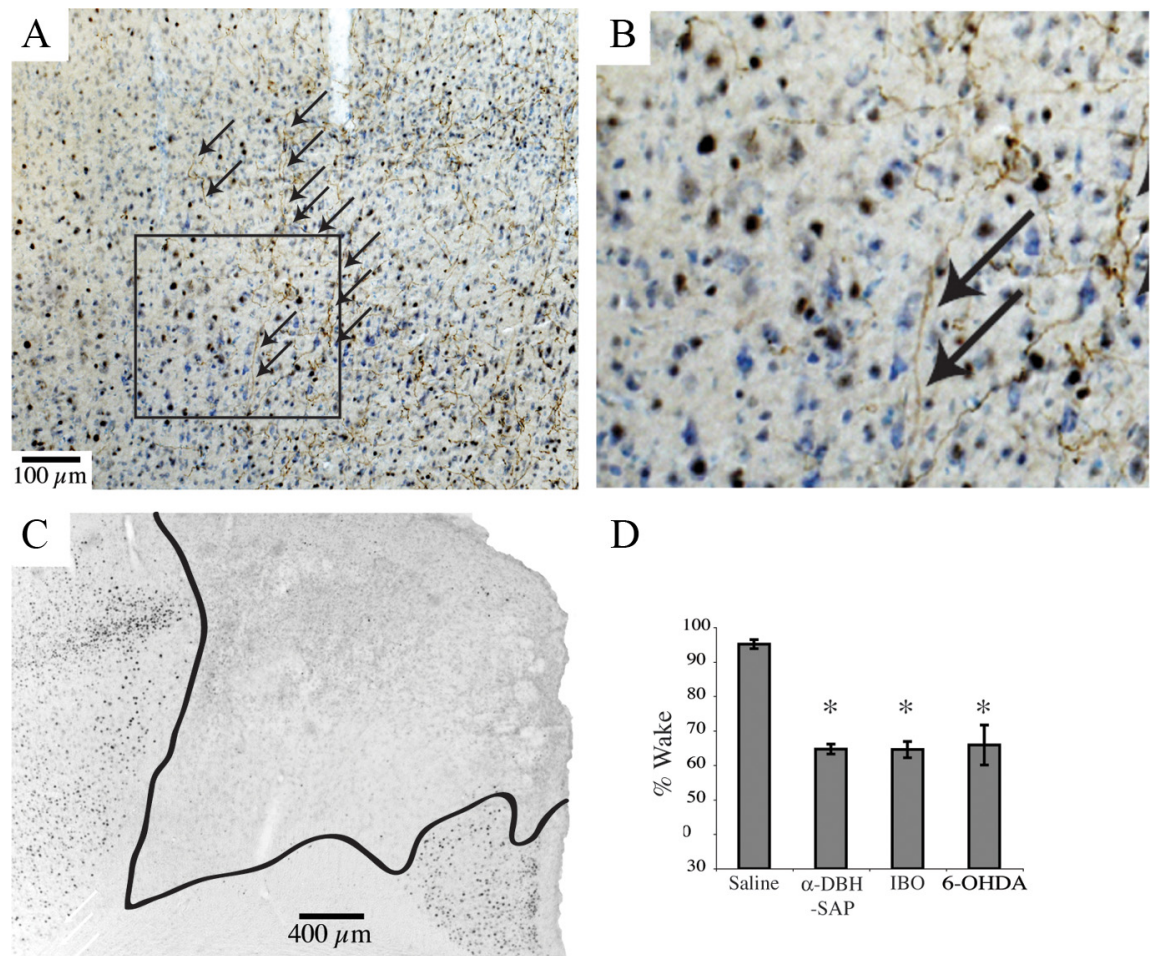

$\mathrm{D}$

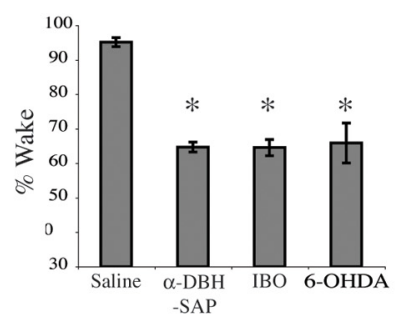

Figure 7. Anterior cingulate cortex involvement in wakefulness induced by a complex environment. $A, A$ photomicrograph showing the border of an injection of 6-OHDA into the ACC (left half) and relatively normal dopamine- $\beta$-hydroxylaseimmunoreactive innervation of the adjacent premotor cortex (fine brown axons on right half, indicated by arrows). The sample was Nissl stained (blue cell bodies) and immunostained for c-Fos (black nuclei). $\boldsymbol{B}$, Boxed area in $\boldsymbol{A}$, magnified to show the transition from lesioned to unlesioned in more detail. $\boldsymbol{C}, \mathrm{ACC}$ lesion (boundaries illustrated by black line) resulting from ibotenate injection into same area as $A$. $D$, For comparison, total wakefulness over the $5 \mathrm{~h}$ period of exposure to the complex environment for animals treated with saline or $\alpha$-DBH-SAP intraventricularly, or with ibotenic acid (IBO) or 6-OHDA injections into the ACC. No significant differences ( ${ }^{*} p=0.9$ ) were found between $\alpha$-DBH-SAP, ibotenic acid, and 6-OHDA-injected animals, indicating that the LC expresses its effects on wakefulness via its inputs to the ACC.

elucidate specific roles for arousal systems that would otherwise not have been found in earlier baseline sleep studies.

The ability of animals and people to maintain alertness in behaviorally critical situations is necessary for survival. The neural mechanisms supporting sustained arousal are therefore likely to come under strong selection pressure and to be similar among mammals. However, traditional baseline sleep/wake recordings have been performed in impoverished and socially isolated environments, largely devoid of stimuli an animal might be expected to interact with in a natural environment. As a result, the role of putative arousal areas in sleep/wake regulation has not been critically examined under naturalistic conditions, and certainly not under conditions in which sustaining arousal is behaviorally required.

In contrast, our observations indicate that, even after allowing time for compensatory responses, the LC is necessary-though not sufficient as it requires cooperation with the ACC-to maintain vigilance under novel behavioral conditions demanding sustained arousal under the adverse circadian phase, even with high homeostatic sleep pressure. Neurophysiologically, LC activity is not only closely correlated with the waking state, but LC firing patterns during wakefulness correlate with the attentional state. In awake animals, LC firing patterns alternate between phasic and tonic patterns when the attentional state varies from scanning to focused, respectively (Aston-Jones and Cohen, 2005). Thus, the functional importance of the LC does not necessarily lie in simply driving the waking state, but rather in the maintenance of arousal when environmental stimuli require attention. This functional role may explain why its loss does not cause much change in baseline wake-sleep when animals are kept in an impoverished environment. Future study will be required to determine whether other arousal areas are also involved in similar wakefulness maintenance under these or other experimental conditions.

An aroused cortex is necessary for attentiveness. The LC sends prominent projections throughout the cerebral cortex, but particularly to the ACC, which it bilaterally innervates. In humans, lesions of the medial prefrontal cortex, including the ACC, produce a syndrome characterized by reduced interest in the environment and inattentiveness (abulia) (Posner et al., 2007). In our rat experiments, we demonstrated the importance of the ACC in maintaining wakefulness in a complex environment. In contrast to studies showing redundancy within the arousal system, we provide evidence for the functional tuning of arousal in specific contexts. It must be mentioned, however, that 6-OHDA injections ablate both LC norepinephrine inputs as well as dopaminergic inputs, which may also contribute to ACC-mediated regulation of sleep-wake states. Although we cannot rule out an independent and overlapping effect of damage to dopamine terminals in this area, the similarity of the effect of the ACC lesions and LC lesions on maintenance of arousal during novelty, and the demonstration of connections between these two areas, strongly suggests that a mutually reinforcing dialog between the LC and the ACC is likely to be a primary mediator of sustained arousal under conditions of behavioral novelty during the normal sleep period. This relationship allows the cortex to maintain executive control over the arousal system.

Consistent with the view that LC inputs play an important role in cortical activation (Cirelli et al., 1996; Cirelli and Tononi, 2000), we found elevated c-Fos expression in the ACC of control animals during active exploration of novel toys, and this c-Fos expression was attenuated in animals that had been mostly asleep after the LC was lesioned. However, Cirelli and coworkers (1996) have reported that unilateral LC lesions caused loss of c-Fos expression throughout the ipsilateral cerebral cortex during wakefulness, suggesting that the LC input may be necessary for cortical neurons to express c-Fos regardless of the state of wakefulness. In contrast, in the conditions in force in our experiments, we found that after either unilateral or bilateral LC lesions there is essentially normal cortical c-Fos expression during sustained wakefulness in the light period, but after $5 \mathrm{~h}$ of novelty stimulation, when bilaterally LC-lesioned animals had slept for much of the last hour, the cortical c-Fos expression was reduced. Thus, the expression of c-Fos in our study was a function of the recent wakefulness of the animals, and did not require intact LC inputs. Instead, our results support the necessity for both an intact LC as well as an intact ACC to override the sleep circuitry that would normally be active during the light phase in rats. Similarly, Cano 

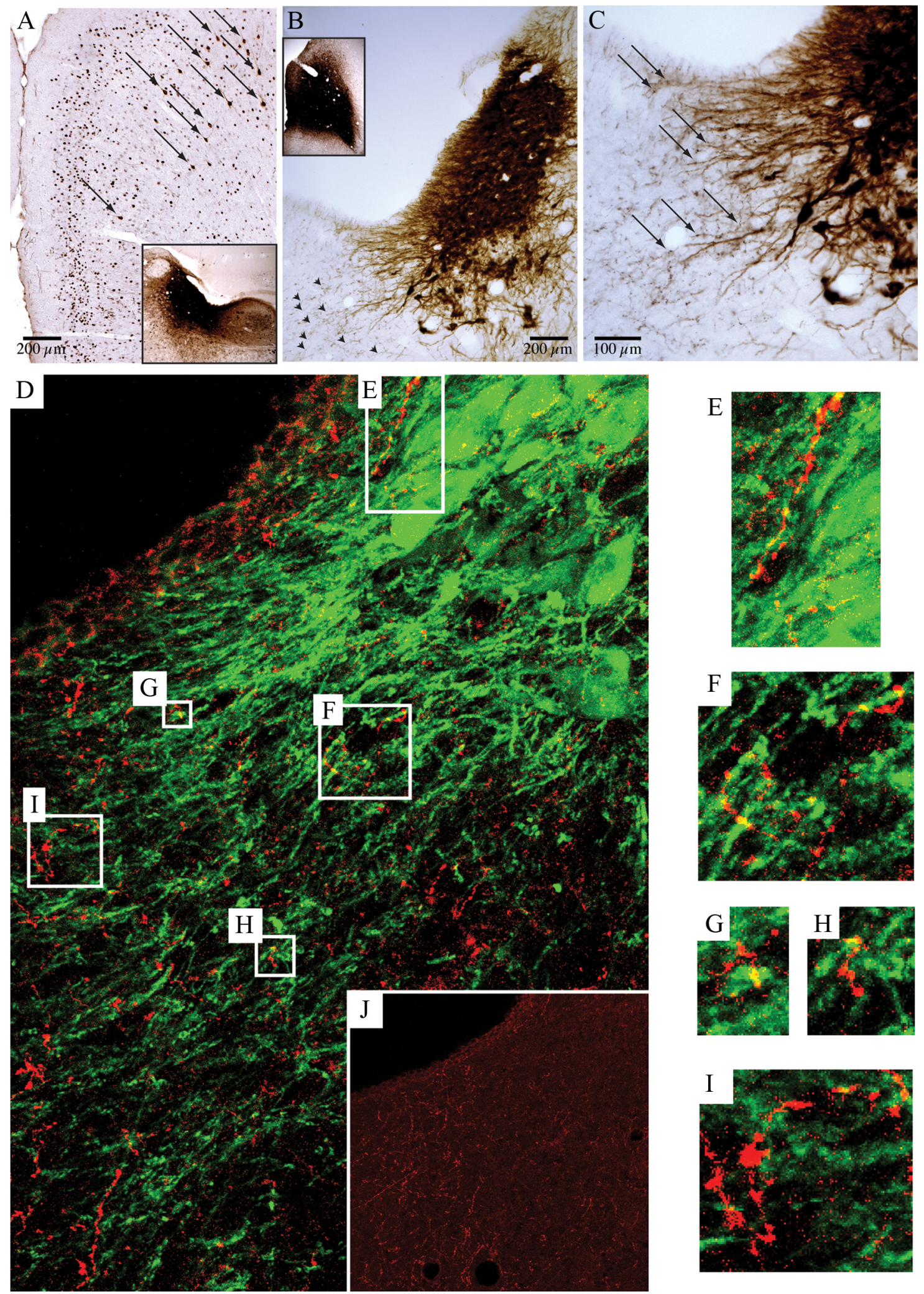

Figure 8. Anterior cingulate cortex provides afferents to the LC. $A$, $C T$ b injected into the $L C$ and the area just medial to it (inset) retrogradely labeled neurons in the $A C C$ (arrows distinguish brown retrogradely labeled neurons from black nuclear Fos staining in the same experiment). $\boldsymbol{B}, \boldsymbol{C}, \mathrm{Low}$ - and high-magnification images of the LC after injection of biotinylated dextranamine into the $A C C$ ( $\boldsymbol{B}$, inset) showed evidence of numerous black axon terminals ( $\boldsymbol{B}$, arrowheads) adjacent to brown TH-immunopositive dendrites projecting medially from the $L C(\boldsymbol{C}$, arrows). $\boldsymbol{D}$, This relationship is best seen with confocal microscopy in an immunofluorescence image from the same case, showing biotinylated dextranamine-stained anterior cingulate axon terminals (red) and tyrosine hydroxylase-positive LC dendrites (green). In this 15 - $\mu \mathrm{m}$-thick $z$-stack composed of twenty $0.75-\mu \mathrm{m}$-thick individual images, boxes correspond to the locations of the enlarged images in $\boldsymbol{E}-\boldsymbol{I}$. Note the yellow areas where overlap between green and red indicates appositions. Appositions were confirmed by examining individual single plane images. A magenta- $\mathrm{green}$ copy of $\boldsymbol{E}-\boldsymbol{J}$ is available as supplemental Figure 1 , available at www.jneurosci.org as supplemental material. $J$, The inset shows the same field as $\boldsymbol{D}$, but with only the red channel to indicate the substantial $A C C$ innervation of this region. 
et al. (2008) recently reported that loss of NREM sleep caused by stress during the light phase was associated with activation of LC and infralimbic cortex, and that lesions of either structure restored normal amounts of NREM sleep.

Generally, medial prefrontal cortex and ACC lesions in rats produce a wider variety of task- and species-specific behavioral impairments than any other cortical lesion (Kolb, 1990) and the LC is activated by medial prefrontal and ACC stimulation (Jodo and Aston-Jones, 1997; Jodo et al., 1998). Our data suggest a model whereby the ACC may not only guide specific behaviors, but, by means of its inputs to the LC, also determine the overall level of arousal of the animal based upon the behavioral context.

\section{References}

Aston-Jones G, Bloom FE (1981) Activity of norepinephrine-containing locus coeruleus neurons in behaving rats anticipates fluctuations in the sleep-waking cycle. J Neurosci 1:876-886.

Aston-Jones G, Cohen JD (2005) An integrative theory of locus coeruleusnorepinephrine function: adaptive gain and optimal performance. Annu Rev Neurosci 28:403-450.

Aston-Jones G, Ennis M, Pieribone VA, Nickell WT, Shipley MT (1986) The brain nucleus locus coeruleus: restricted afferent control of a broad efferent network. Science 234:734-737.

Berridge CW, España RA (2000) Synergistic sedative effects of noradrenergic alpha(1)- and beta-receptor blockade on forebrain electroencephalographic and behavioral indices. Neuroscience 99:495-505.

Berridge CW, Foote SL (1991) Effects of locus coeruleus activation on electroencephalographic activity in neocortex and hippocampus. J Neurosci 11:3135-3145.

Berridge CW, Waterhouse BD (2003) The locus coeruleus-noradrenergic system: modulation of behavioral state and state-dependent cognitive processes. Brain Res Brain Res Rev 42:33-84.

Berridge CW, Page ME, Valentino RJ, Foote SL (1993) Effects of locus coeruleus inactivation on electroencephalographic activity in neocortex and hippocampus. Neuroscience 55:381-393.

Blanco-Centurion C, Gerashchenko D, Shiromani PJ (2007) Effects of saporin-induced lesions of three arousal populations on daily levels of sleep and wake. J Neurosci 27:14041-14048.

Cano G, Mochizuki T, Saper CB (2008) Neural circuitry of stress-induced insomnia in rats. J Neurosci 28:10167-10184.

Cedarbaum JM, Aghajanian GK (1978) Afferent projections to the rat locus coeruleus as determined by a retrograde tracing technique. J Comp Neurol 178:1-16.

Cirelli C, Tononi G (2000) Differential expression of plasticity-related genes in waking and sleep and their regulation by the noradrenergic system. J Neurosci 20:9187-9194

Cirelli C, Pompeiano M, Tononi G (1996) Neuronal gene expression in the waking state: a role for the locus coeruleus. Science 274:1211-1215.

Foote SL, Berridge CW, Adams LM, Pineda JA (1991) Electrophysiological evidence for the involvement of the locus coeruleus in alerting, orienting, and attending. Prog Brain Res 88:521-532.

Gaus SE, Strecker RE, Tate BA, Parker RA, Saper CB (2002) Ventrolateral preoptic nucleus contains sleep-active, galaninergic neurons in multiple mammalian species. Neuroscience 115:285-294.

González MM, Aston-Jones G (2006) Circadian regulation of arousal: role of the noradrenergic locus coeruleus system and light exposure. Sleep 29:1327-1336.

González MM, Debilly G, Valatx JL (1998) Noradrenaline neurotoxin DSP-4 effects on sleep and brain temperature in the rat. Neurosci Lett 248:93-96.

Hurley KM, Herbert H, Moga MM, Saper CB (1991) Efferent projections of the infralimbic cortex of the rat. J Comp Neurol 308:249-276.

Jodo E, Aston-Jones G (1997) Activation of locus coeruleus by prefrontal cortex is mediated by excitatory amino acid inputs. Brain Res 768: 327-332.

Jodo E, Chiang C, Aston-Jones G (1998) Potent excitatory influence of pre- frontal cortex activity on noradrenergic locus coeruleus neurons. Neuroscience 83:63-79.

Kolb B (1990) Prefrontal cortex. In: The cerebral cortex of the rat (Kolb B, Tees RC, ed), pp 437-458. Cambridge, MA: MIT.

Léger L, Goutagny R, Sapin E, Salvert D, Fort P, Luppi PH (2009) Noradrenergic neurons expressing Fos during waking and paradoxical sleep deprivation in the rat. J Chem Neuroanat 37:149-157.

Lidbrink P (1974) The effect of lesions of ascending noradrenaline pathways on sleep and waking in the rat. Brain Res 74:19-40.

Lu J, Bjorkum AA, Xu M, Gaus SE, Shiromani PJ, Saper CB (2002) Selective activation of the extended ventrolateral preoptic nucleus during rapid eye movement sleep. J Neurosci 22:4568-4576.

Lu J, Jhou TC, Saper CB (2006) Identification of wake-active dopaminergic neurons in the ventral periaqueductal gray matter. J Neurosci 26:193-202.

Lu J, Nelson LE, Franks N, Maze M, Chamberlin NL, Saper CB (2008) Role of endogenous sleep-wake and analgesic systems in anesthesia. J Comp Neurol 508:648-662.

Luppi PH, Aston-Jones G, Akaoka H, Chouvet G, Jouvet M (1995) Afferent projections to the rat locus coeruleus demonstrated by retrograde and anterograde tracing with cholera-toxin B subunit and Phaseolus vulgaris leucoagglutinin. Neuroscience 65:119-160.

Matsuyama S, Coindet J, Mouret J (1973) Intracisternal 6-hydroxydopamine and sleep in the rat. Brain Res 57:85-95.

Monti JM, D’Angelo L, Jantos H, Barbeito L, Abó V (1988) Effect of DSP-4, a noradrenergic neurotoxin, on sleep and wakefulness and sensitivity to drugs acting on adrenergic receptors in the rat. Sleep 11:370-377.

Nithianantharajah J, Hannan AJ (2006) Enriched environments, experiencedependent plasticity and disorders of the nervous system. Nat Rev Neurosci 7:697-709.

Ouyang M, Hellman K, Abel T, Thomas SA (2004) Adrenergic signaling plays a critical role in the maintenance of waking and in the regulation of REM sleep. J Neurophysiol 92:2071-2082.

Paxinos G, Watson C (1998) The rat brain in stereotaxic coordinates, Ed 4. San Diego: Academic.

Posner J.B., Saper C.B., Schiff N., Plum F. (2007) Plum and Posner's diagnosis of stupor and coma, 4th Ed. New York: Oxford UP.

Roussel B, Pujol JF, Jouvet M (1976) Effects of lesions in the pontine tegmentum on the sleep stages in the rat (in Italian). Arch Ital Biol 114:188-209.

Saper CB, Scammell TE, Lu J (2005) Hypothalamic regulation of sleep and circadian rhythms. Nature 437:1257-1263.

Sara SJ, Dyon-Laurent C, Hervé A (1995) Novelty seeking behavior in the rat is dependent upon the integrity of the noradrenergic system. Brain Res Cogn Brain Res 2:181-187.

Sauer H, Oertel WH (1994) Progressive degeneration of nigrostriatal dopamine neurons following intrastriatal terminal lesions with 6-hydroxydopamine: a combined retrograde tracing and immunocytochemical study in the rat. Neuroscience 59:401-415.

Swanson LW (1976) The locus coeruleus: a cytoarchitectonic, Golgi and immunohistochemical study in the albino rat. Brain Res 110:39-56.

Takada M, Campbell KJ, Moriizumi T, Hattori T (1990) On the origin of the dopaminergic innervation of the paraventricular thalamic nucleus. Neurosci Lett 115:33-36.

Takahashi K, Kayama Y, Lin JS, Sakai K (2010) Locus coeruleus neuronal activity during the sleep-waking cycle in mice. Neuroscience 169: $1115-1126$.

Van Bockstaele EJ, Chan J, Pickel VM (1996) Input from central nucleus of the amygdala efferents to pericoerulear dendrites, some of which contain tyrosine hydroxylase immunoreactivity. J Neurosci Res 45:289-302.

van Praag H, Kempermann G, Gage FH (2000) Neural consequences of environmental enrichment. Nat Rev Neurosci 1:191-198.

Vankov A, Hervé-Minvielle A, Sara SJ (1995) Response to novelty and its rapid habituation in locus coeruleus neurons of the freely exploring rat. Eur J Neurosci 7:1180-1187.

Wrenn CC, Picklo MJ, Lappi DA, Robertson D, Wiley RG (1996) Central noradrenergic lesioning using anti-DBH-saporin: anatomical findings. Brain Res 740:175-184. 